\title{
Machine Learning for Affective Computing
}

\author{
Mohammed Hoque ${ }^{1}$, Daniel J. McDuff ${ }^{1}$, Louis-Philippe Morency ${ }^{2}$, \\ and Rosalind W. Picard ${ }^{1}$ \\ ${ }^{1}$ MIT Media Lab, Cambridge, MA 02139, USA \\ \{mehoque, djmcduff, picard\} amedia.mit.edu \\ ${ }^{2}$ Institute for Creative Technologies, University of Southern California, \\ Marina del Ray, CA 90292, USA \\ morency@ict.usc.edu
}

\begin{abstract}
Affective computing (AC) is a unique discipline which includes modeling affect using one or multiple modalities by drawing on techniques from many different fields. AC often deals with problems that are known to be very complex and multi-dimensional, involving different kinds of data (numeric, symbolic, visual etc.). However, with the advancement of machine learning techniques, a lot of those problems are now becoming more tractable.

The purpose of this workshop was to engage the machine learning and affective computing communities towards solving problems related to understanding and modeling social affective behaviors. We welcomed participation of researchers from diverse fields, including signal processing and pattern recognition, statistical machine learning, human-computer interaction, human-robot interaction, robotics, conversational agents, experimental psychology, and decision making.

There is a need for a set of high standards for recognizing and understanding affect. At the same time, these standards need to take into account that the expectations and validations in this area may be different than in traditional research on machine learning. This should be reflected in the design of machine learning techniques used to tackle these problems. For example, affective data sets are known to be noisy, high dimensional, and incomplete. Classes may overlap. Affective behaviors are often person specific and require temporal modeling with real-time performance. This first edition of the ACII Workshop on Machine Learning for Affective Computing will be a proper venue to invoke such discussions and engage the community towards design and validation of learning techniques for affective computing.
\end{abstract}

Keywords: spontaneous affective states, automated recognition of affect, multimodal data fusion, probabilistic modeling, feature selection. 